\title{
Expectation-maximization-based channel estimation algorithm for OFDM visible light communication systems
}

\author{
Yaseein Soubhi Hussein ${ }^{1}$, Mohamad Yusoff Alias ${ }^{2}$, Ayad A. Abdulkafi ${ }^{3}$, Nazaruddin Omar ${ }^{4}$, \\ Mohd Kamarulzamin Bin Salleh ${ }^{4}$ \\ ${ }^{I}$ Faculty of Computing, Engineering \& Technology (FCET), Asia Pacific University of Technology \& Innovation, 57000 Kuala Lumpur, \\ Kuala Lumpur, Malaysia \\ ${ }^{2}$ Faculty of Engineering, Multimedia University, 63100 Cyberjaya, Selangor, Malaysia \\ ${ }^{3}$ College of Applied Engineering, Tikrit University, Iraq \\ ${ }^{4}$ TM Research \& Development Sdn Bhd, Innovation Centre, Lingkaran Teknokrat Timur, 63000 Cyberjaya Selangor, Malaysia \\ *Corresponding author E-mail: dr.yaseein@apu.edu.my
}

\begin{abstract}
The tremendous growth of indoor communication requires increased capacity and appropriate quality of services. Visible light communica-tion (VLC) is a green technology that shows great promise in terms of its ability to meet the demand for communication services. Orthogo-nal frequency division multiplexing (OFDM) enables VLC to provide a higher data rate and to combat inter-symbol interference. However, an accurate and efficient channel estimation method is needed for coherent demodulation at the receiver end of an OFDM system. In this paper, a new algorithm for OFDM-based VLC systems is proposed. The algorithm is based on expectation maximization and is called the expectation maximization for visible light communication (EM-VLC) algorithm. The algorithm is implemented to find the maximum-likelihood (ML) estimation of the channel impulse response and to find unknown parameters. In addition, a low-rank minimum mean square error (lr-MMSE) estimator algorithm is developed and its performance is compared with least squares (LS) and minimum mean square error (MMSE) estimators. The proposed EM-VLC algorithm improves the performance of OFDM VLC systems by significantly reducing the bit error rate (BER) and consequently increasing system throughput. The simulation results demonstrate that the EM-VLC algorithm outper-forms the three channel estimation algorithms, LS, MMSE and lr-MMSE.
\end{abstract}

Keywords: Bit Error Rate; Channel Estimation; Expectation Maximization; Optical OFDM Visible Light Communication.

\section{Introduction}

Visible light communication (VLC) is a viable technology that possesses many attractive qualities, such as an unlicensed spectrum, worldwide availability of unused bandwidth, noninterference with radio frequency (RF) bands, very high data rates and secure communication [1], [2]. Visible light communication uses low-cost light-emitting diodes (LEDs) as transmitters, or a converter, to translate the modulated electrical signal into an optical signal. Then, a reverse process allows the photodiodes (PDs) to detect the signal and demodulate it via a digital signal processing technique [3]. Orthogonal frequency division multiplexing (OFDM) in VLC holds promise as a means of providing a higher data rate, combating inter-symbol interference (ISI) and reducing multipath effects. However, the nonlinearities of the OFDM transmitted signal and the wireless optical propagation effects increase the complexity involved in retrieving the original signal at the receiver. Hence an accurate and efficient channel estimation technique is needed. Channel estimation refers to the estimation of the channel effect on the transmitted signal and the compensation process that is used to recover the transmitted signal at the receiver. Channel estimation techniques can be categorized into 1) training sequence methods (also known as block-type pilot arrangements), which introduce pilot tones into the subcarriers of the OFDM symbols [4], 2) blind channel methods, which exploit the statistical or structural properties of communication signals, such as the frequency correlation and cyclic prefix (CP) (i.e., they do not use a training sequence); and 3) semi-blind channel methods, which are based on a combination of blind channel and training sequence methods and which use pilot arrangements and the structural characteristics of the signals [5].

In this paper, a new channel estimation method is developed in an attempt to reduce the bit error rate (BER) and the analytical of complexity in direct current optical OFDM (DCO-OFDM). The remainder of the paper is organized as follows: in section 2, related works on channel estimation in OFDM-based VLC are introduced. In section 3, the OFDM VLC system and channel estimation are described. In section 4, the proposed expectation maximization for visible light communication (EM-VLC) algorithm for OFDM VLC systems is presented and analysed. In section 5 , the simulation results are presented and discussed. In section 6 , a conclusion is provided.

\section{Related work}

A few approaches, such as those in [6 - 8], have been proposed for channel estimation in OFDM-based VLC systems. The primary objective of these approaches, as well as that of a more recent work [9], is to reduce the BER and thus obtain better system performance. Several other channel estimation methods have also been proposed, such as those in [10 - 14]. However, these are for optical wireless communication (OWC) systems rather than 
OFDM. The channel estimation methods in OWC are trainingsequence-based and can be performed by utilizing least squares (LS) or minimum mean square error (MMSE) algorithms [15] as well as other modified MMSE algorithms, such as low-rank minimum mean square error (lr-MMSE) algorithms [16]. Furthermore, some other methods that have been proposed for purposes other than channel estimation could also be used for channel estimation. These methods, which were proposed in [17] and [18], aimed to overcome the nonlinearities in OFDM VLC systems, including the peak to average power ratio (PAPR).

In [6], a method that combines discrete Fourier transformation (DFT) with post-processing and LS, called DFT-LS, was proposed for DCO-OFDM with the aim of improving system performance by eliminating the noise beyond the maximum delay of the channel. The method uses a LS approach for channel estimation with a comb-type pilot arrangement and linear interpolation. While DFTLS performs better than the ordinary LS estimator in terms of BER at different orders of constellation, it is less accurate than an MMSE estimator is. [19].

In [7], an adaptive channel estimation method based on a LS discrete Fourier transform (LS-DFT) and orthogonal matching pursuit (OMP) was proposed as a means to enhance the reliability of asymmetrically clipped DC-biased optical (ACO-OFDM) VLC systems. The first part of the algorithm is similar to that proposed in [6] in which a post-process DFT-LS/LS-DFT is used to obtain the channel response in the frequency domain after eliminating the noise effect [16]. The second part of the algorithm is an iterative greedy OMP algorithm that is used to obtain the channel response in the time domain. The results show that LS-DFT achieves a better BER than the DFT-LS presented in [6]. However, an additional computational cost is incurred. The results of an investigation into the differences between DCO-OFDM and ACO-OFDM in VLC systems can be found in [20].

In [8], an adaptive and specific grid-type pilot arrangement was proposed as a way to evaluate the channel response in DCOOFDM VLC systems. This method is based on sending known subcarriers from the transmitter in one or more OFDM symbols to the receiver. At the receiver, the channel response can be found by estimating the subcarrier changes. The use of more pilots can improve channel estimation, but such an approach sacrifices transmission capacity. However, in this method, fewer pilot insertions are needed, so it has better transmission capacity than methods that use training symbols (block-type pilots). Moreover, this method can adapt the pilot tones according to the BER performance, at the cost, the signal-to-noise ratio (SNR) cannot maintain the transmission. In [9], a more recent work, an adaptive statistical channel estimation of Bayesian MMSE was proposed in which the variable statistic window is exploited, but this approach has high computational complexity.

In this paper, a new algorithm for OFDM-based VLC systems is proposed. It is based on EM and is called the expectation maximization for visible light communication (EM-VLC) algorithm. In addition, a lr-MMSE algorithm is developed for OFDM VLC systems. The proposed EM-VLC algorithm is an iterative method for computing the maximum-likelihood (ML) channel estimation and finding unknown parameters. It consists of two steps: the first step involves expectation (E) for unknown parameters and the second step involves maximization (M) to maximize the expectation of the log-likelihood function. The EM algorithm has been widely used in RF for channel estimation, for instance in [21 - 25]. However, EM for indoor VLC is different from that for conventional RF due to several limitations, and it is these limitations that motivated the present study. The simulation results of the proposed algorithm demonstrate its ability to significantly reduce the BER. Moreover, it is a powerful channel estimation solution for OFDM VLC systems.

\section{The system model}

\subsection{The OFDM VLC system}

In this section, the OFDM-based VLC system is presented together with its channel model. The OFDM VLC system is illustrated in Fig. 1. The typical OFDM signal is bipolar and complex, so it is impossible to transmit such a signal via an optical wireless channel using intensity modulated/direct detection (IM/DD). In VLC, the signal must be unipolar and real. Thus, Hermitian symmetry in the inverse fast Fourier transform (IFFT) and a DC-bias current are needed to make the signal real and positive, respectively. This is referred to as the DC-biased optical OFDM (DCO-OFDM) scheme [26].

In the VLC system, a LED serves as a transmitter and a PD acts as a receiver. The number of multipath components is large and the propagation channel varies temporally due to the movement of users and other natural propagation processes of light, such as reflection and diffraction. The noise due to background light can be exhibited as additive white Gaussian noise (AWGN) within the model. There is no interference with other RF communication systems [27].

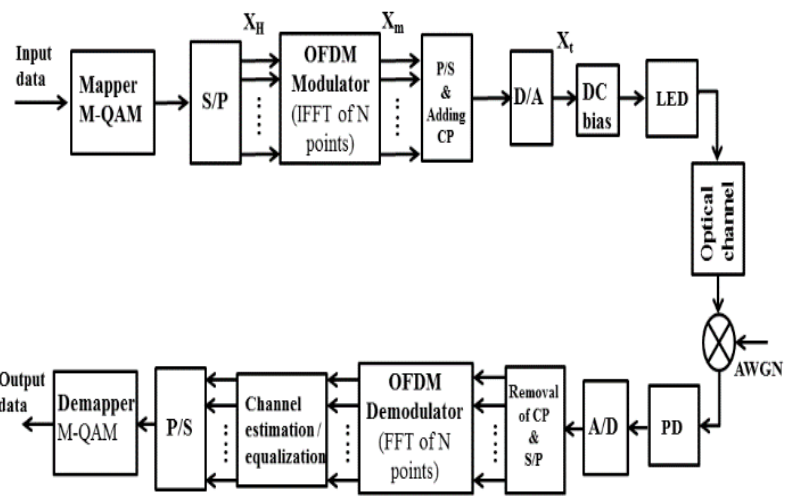

Fig. 1: Block Diagram of DCO-OFDM VLC System.

At the transmitter end of the system, the mapping to an M-QAM constellation has to be done before the Hermitian symmetry, $\mathrm{X}_{\mathrm{H}}$, is applied. The IFFT operation results in $2 \mathrm{~N}$ points in the frequency domain. Then, the modulated OFDM symbol vector, $\mathrm{x}$, in the time domain, $\mathrm{x}_{\mathrm{m}}$, is converted from parallel to serial $(\mathrm{P} / \mathrm{S})$, adding the $\mathrm{CP}$ and digital-to-analogue (D/A) conversion, as shown in Equation (1):

$x_{m}=\frac{1}{N} \sum_{h=0}^{2 N-1} X_{H, h} e^{j \frac{2 \pi h m}{N}} \quad$ for $0 \leq m \leq 2 N-1$

Where $h$ is the $h^{\text {th }}$-subcarrier symbol of $X_{H}$.

The bipolar and real value of OFDM, $x_{t}$, is obtained in the time domain. Then, the DC bias is applied to obtain a new unipolar or positive signal as an input to the LED. Finally, the signal derives the LED that can convert the electrical signal into an optical intensity. With the PD acting as a receiver, the signal can be directly detected. The reverse process is carried out in addition to channel estimation to retrieve the original transmitted signal. The output received signal of the channel can be expressed as:

$$
y(n)=x(n) \otimes h(n)+w(n)
$$

Where $\mathrm{h}(\mathrm{n})$ is the channel impulse response. It is assumed that the channel is linear and $2 \mathrm{~N}$ is the received time-domain signal length. An AWGN channel is represented by w (n).

Then, the output in the frequency domain is obtained, in which the FFT of $\mathrm{h}(\mathrm{n})$ and $\mathrm{w}(\mathrm{n})$ in Equation (2) becomes $\mathrm{H}(\mathrm{k})$ and $\mathrm{W}(\mathrm{k})$ in Equation (3), respectively, as follows:

$$
Y(k)=X(k) H(k)+W(k) \quad k=0, \ldots, N-1
$$

\subsection{Channel estimation}


Channel estimation is the key feature of the OFDM VLC system. The received signal is influenced by the channel characteristics and an accurate channel estimation is needed to recover the original transmitted bits [28]. Thus, channel estimation is an essential part of OFDM systems. A good channel estimation algorithm should have both low complexity and high accuracy. Generally, two common techniques can be employed for channel estimation. The first involves using training symbols when pilots (known information) are sent when no data is being sent. The second involves using pilot aided where the pilot inserted together with the data. In the frequency domain, the pilot spacing depends on the frequency coherence, which is related to the delay spread, so it must be determined carefully [29].

In the VLC system, the shadowing effect can be alleviated by using numerous LEDs. However, increasing the number of LEDs aggravates the multipath effect, which leads to ISI. Inter-symbol interference is a significant issue in OFDM VLC systems because it can reduce the system's data rate as well as its BER [30]. Hence channel estimation is a critical issue and achieving coherent demodulation is a challenging task. The two common channel estimation methods for OFDM VLC systems - LS and MMSE - are described below. Then, the lr-MMSE estimator developed for this work is presented.

\subsubsection{Least squares}

The main reason for using the LS algorithm is to find an optimal estimator for the unknown parameters by minimizing the LS errors [31]. Generally, LS is used to get an initial channel estimation at the pilot subcarriers [32]. The channel statistical model in the frequency domain for the OFDM VLC system can be modelled as

$Y=\bar{X} H+W$

Where the Gaussian noise is denoted by $\mathrm{W}$ with variance $\sigma 2$.

Then, the input matrix is $\bar{X}=\operatorname{diag}\left(\left\{X_{\mathrm{k}}\right\}\right)$. To find the LS channel estimation, the sum of the square errors of channel estimation is computed in the frequency domain. By using differentiation and setting Equation (4) to zero with each channel coefficient $\mathrm{H}$, the LS channel estimation in the frequency domain can be calculated as follows:

$\widehat{H}_{L S}=\frac{Y}{X}$

The channel estimation objective and estimation error can be found by substituting Equation (4) into Equation (5):

$$
\widehat{H}_{L S}=H+\frac{W}{X}
$$

Where $\mathrm{W} / \mathrm{X}$ is the estimated error and $\mathrm{H}$ is the objective of channel estimation. The LS is simple and easy to implement. However, it has low accuracy.

\subsubsection{Minimum mean square error}

The main aim of using the MMSE algorithm is to develop an optimal estimator for finding the unknown parameters by minimizing the mean square error (MSE). Defining the linear estimator operator L [16] finds the MMSE as

$$
\widehat{H}_{M M S E}=L Y
$$

Based on Equation (7), the MSE of the channel estimation for the OFDM VLC system can be modelled as

$$
\partial(L)=\mathrm{E}\left[\left\|\left(H-\hat{H}_{M M S E}\right)\right\|^{2}\right]
$$

Where E [•] is the expectation operator. First, it is expected that to determine the estimator value $\mathrm{L}$, to obtain the MMSE channel estimation. Therefore, the value of $\mathrm{L}$ must be satisfied by Equation (9) below:

$$
\widehat{L}=\arg \min _{L} \partial(L)
$$

In a similar manner to LS, MMSE uses differentiation and sets to zero with respect to each channel coefficient $\mathrm{L}$, as below:

$$
\frac{\partial}{\partial L} \mathrm{E}\left[(H-L Y)(H-L Y)^{H}\right]=0
$$

By solving Equation (10), the value of operator L can be obtained as

$$
\widehat{L}=\frac{R_{H} \bar{X}^{H}}{\bar{X} R_{H} \bar{X}^{H}+R_{W}}
$$

Where $R_{W}=E[W W H]$ is the covariance matrix of noise, $R_{H}=E$ $[\mathrm{HHH}]$ is the covariance matrix of the channel coefficient in the frequency domain and $\sigma^{2}$ is the noise variance. Thus, the MMSE channel estimation can be expressed as

$$
\begin{gathered}
\hat{H}_{M M S E}=\widehat{L} Y=\left[\frac{R_{H} \bar{X}^{H}}{\bar{X} R_{H} \bar{X}^{H}+R_{W}}\right] Y \\
=R_{H}\left[\frac{1}{\left(R_{H}+\sigma^{2}\left(\frac{1}{\overline{X X}}\right)\right)}\right] \hat{H}_{L S}
\end{gathered}
$$

From Equation (12), it is can be seen that the MMSE channel estimation method mitigates the channel noise effects relatively better than the LS method. However, in the MMSE method more prior knowledge is needed about the channel coefficient matrix (auto-covariance) and noise covariance matrix, $\mathrm{R}_{\mathrm{H}}$ and $\mathrm{R}_{\mathrm{W}}$, respectively. In practice, these unknown parameters of channel frequency response make MMSE channel estimation almost impossible in real time [29]. Therefore, the MMSE method has greater complexity than the LS method.

\subsubsection{Low-rank minimum mean square error}

The low-rank approximation for MMSE channel estimation developed for this paper simplifies the computational process of MMSE to reduce complexity in three ways. The first simplification involves replacing $\left(\overline{X X}^{H}\right)^{-1}$ by its expectation $E=\left\{\left(\overline{X X}{ }^{H}\right)^{-1}\right\}$. It is assumed that the constellations of the signal on all tones are the same and that there is an equal probability on all constellation points, thus:

$\left.E=\left\{(\overline{X X})^{H}\right)^{-1}\right\}=E\left\{1 / X_{k}\right\}^{2} I$

Where I represents the identity matrix.

This defines the mean SNR as

$\overline{S N R}=E\left\{\left|X_{k}\right|^{2}\right\} / \sigma^{2}$

Then, using ${ }^{\left.\beta=E \|\left. X_{k}\right|^{2}\right\} E\left\{1 / x_{k}\right\}^{2}}$, the MMSE channel estimation can be rewritten as

$\left.\hat{H}_{\text {MMSE }}=R_{H}\left[\frac{1}{\left(R_{H}+\left(\frac{\beta}{S N R}\right) I\right.}\right)\right] \hat{H}_{L S}$

The singular value decomposition (SVD) is performed over Hermitian $R_{H}$ : 
$R_{H}=U \Lambda U^{H}$

Where $\mathrm{U}$ denotes a unitary matrix that contains singular vectors and $\Lambda$ is referred to as a diagonal matrix that contains singular values that are $\lambda 1 \geq \lambda 2 \geq \cdots \geq \lambda \mathrm{M}$ on its diagonal.

The use of SVD significantly decreases the complexity of the calculation process of the matrix. First, the estimator finds the required number of ranks, denoted by $\mathrm{p}$ in Equation (17), which should be bigger than $(\mathrm{J}+1)$ [33]. Then, the rest of the parameters that appear in Equation (17) are as given; the singular values, $\lambda \mathrm{k}$, the channel auto-covariance matrix, $\mathrm{RH}$, the noise variance, SNR, the receiver pre-calculates, $\beta$, and the unitary matrix, $U$.

In this paper, the lr-MMSE channel estimation algorithm was developed so that it would be compatible with the OFDM VLC system. Therefore, $\beta$ is a constant and must be divided by 2 due to its unipolar signal and it depends only on the signal constellation. Therefore, the $\mathrm{M} \times \mathrm{M}$ diagonal matrix, ${ }^{\Delta_{p}}$, is obtained with the following parameters:

$$
\delta_{k}=\left\{\begin{aligned}
{\left[\frac{\lambda_{k}}{\lambda_{k}+\frac{\beta / 2}{S N R}}\right], } & k=0,1, \ldots, p-1 \\
0 \quad & k=p, \ldots . M-1
\end{aligned}\right.
$$

To calculate the LS according to Equation (4), the pilots $X$ and received signals $\mathrm{Y}$ are used during transmission, where the $\mathrm{lr}$ MMSE channel estimation with the rank $\mathrm{p}$ is given by

$$
\widehat{H}_{l r-M M S E}=U \Delta_{p} U^{H} \hat{H}_{L S}
$$

From Equations (17) and (18), it can be seen that the adapted lrMMSE estimator proposed above is significantly less complex than the MMSE estimator and also performs better at low SNR values. The reduction in the complexity of channel estimation provided by the proposed lr-MMSE is due to the optimal value of the subspace dimension within $\mathrm{J}+1$, and maintain defining the channel as well [34], compared to the conventional Ir-MMSE estimator where there is a problem of recovering the Ir-MMSE matrix and uncertainty quantification [35].

\section{Proposed EM-VLC algorithm}

The new channel estimation method for OFDM VLC systems proposed in this paper is a semi-blind technique that uses an iterative method [28] to determine the ML estimation of the channel. This iterative method of channel estimation can be employed to reduce the channel estimation errors. The greater the number of iterations, the better the performance achieved. However, this results in a greater computational cost. To mitigate this drawback, the proposed EM-VLC algorithm uses the same error probabilities that have already been used by the decoders. For instance, in turbo decoding, the maximum a posteriori decoder $[15,36]$ is utilized to find the expectations of the symbols to be transmitted. This is exactly the approach that has been adopted in the proposed EMVLC algorithm for channel estimation. Thus, the computational complexity of the EM-VLC algorithm is reduced significantly. Moreover, no prior knowledge of the channel condition is required. The proposed algorithm is an iterative method for computing the ML channel estimation and finding unknown parameters. It is executed in two steps. The first step involves expectation (E) for unknown parameters and the second step involves maximization (M) to maximize the expectation of the log-likelihood function. These two steps are repeated until the estimated values converge [37].

Thus, it is assumed that $\mathrm{X}$ stands for one of the M-ary symbols in the frequency domain of the constellation size $\mathrm{M}$, in which $\mathrm{X} \in$ $\{\mathrm{X} 1, \mathrm{X} 2, \ldots . \mathrm{XM}\}$, where $\mathrm{Xi}$ refers to the ith symbol of the con- stellation. According to the received signal in Equation (4), the conditional probability density function (PDF) of output $\mathrm{Y}$ given $\mathrm{X}$ and $\mathrm{H}$ is as follows:

$$
f(Y \mid X, H)=\frac{1}{2 \pi \sigma^{2}} \exp \left(-\frac{1}{2 \sigma^{2}}|Y-H X|^{2}\right)
$$

Next, Gaussian random variables with variance $\sigma 2$ and zero mean are considered. Then, assuming that the probability and averaging of all $\mathrm{M}$ symbols in the constellation are equal for the conditional PDF in Equation (19), the received signal can be expressed Y given $\mathrm{H}$ over $\mathrm{X}$ as follows:

$$
f(Y \mid H)=\sum_{i=1}^{M} \frac{1}{M} \frac{1}{2 \pi \sigma^{2}} \exp \left(-\frac{1}{2 \sigma^{2}}\left|Y-H \cdot X_{i}\right|^{2}\right)
$$

For indoor VLC, assuming the channel is not time dependent over a D of OFDM symbols, the transmitted and received symbols in the vector can be denoted as $\bar{Y}=[\mathrm{Y} 1, \mathrm{Y} 2, \ldots \mathrm{YD}] \mathrm{T}$ and $\bar{X}=$ $[\mathrm{X} 1, \mathrm{X} 2, \ldots \mathrm{XD}] \mathrm{T}$, respectively.

The objective in this work is to find the estimated value of the transmitted bits, $\bar{X}$ from the received signal, $\bar{Y}$ as expressed in Equation (4), and to reduce the BER caused by the wireless optical channel. The EM-VLC algorithm is used to estimate $\bar{X}$ and maximize $f(\bar{Y} \mid \bar{X})$ by calculating the mean of the log-likelihood function $f(\bar{Y}, \bar{h} \mid \bar{X})$ over the unknown parameters $\bar{h}$. Therefore, it is essential to determine the channel frequency response $\mathrm{H}$ in order to estimate the transmitted signal X. Here, ${ }^{(\bar{Y}, \bar{h})}$ represents complete data and ${ }^{(\bar{Y})}$ denotes incomplete data. It is not easy to expect the channel frequency response based on the 'incomplete' data. However, the PDF of 'incomplete' data can be converted into the PDF of 'complete' data, and expressed as

$$
f(\bar{Y} \mid H, \bar{X})=\prod_{d=1}^{D} f\left(Y^{d} \mid H, X^{d}\right)
$$

Therefore, the incomplete data log-likelihood function is given by

$\log f(\bar{Y} \mid H, \bar{X})=\sum_{d=1}^{D} \log f\left(Y^{d} \mid H, X^{d}\right)$

The PDF of 'complete' data can be written in the same manner as in Equation (22) by using the log-likelihood function as follows:

$$
\log f(\bar{Y}, \bar{X} \mid H)=\sum_{d=1}^{D}\left(\log \frac{1}{M} f\left(Y^{d} \mid H, X^{d}\right)\right)
$$

In each iterative process of $\mathrm{p}$, where $\mathrm{p}$ can be any integer number $(0,1,2),, \mathrm{H}$ can be estimated by maximizing $f(\bar{Y} \mid H)$ and by iteratively increasing the likelihood function at each step.

The EM-VLC algorithm consists of the following two steps to estimate $\mathrm{H}$ from $\bar{Y}$ :

Expectation (E):

$$
Q\left(H \mid H_{p}\right)=E\left\{\log f(\bar{Y}, \bar{X} \mid H) \mid \bar{Y}, H_{p}\right\}
$$

Maximization (M):

$H_{p+1}=\arg \max _{H} Q\left(H \mid H_{p}\right)$

In the E step, Hp represents the latest estimate of $\mathrm{H}$. The estimated value of the log-likelihood function of $\mathrm{H}$ is calculated by taking the estimation over $\bar{X}$, considering the value of $\bar{Y}$ and using the most recent estimated value of $H$. In the subsequent $M$ step, $\mathrm{Hp}+1$ is determined as in Equation (25). The final estimation of $(p+1) s t$ of $\mathrm{H}$ becomes $\mathrm{H}(\mathrm{p}+1)$, which is the additional manipulation of $\mathrm{H}(\mathrm{p}+1)$. These two steps should result in a sufficiently small difference in value between $H(p+1)$ and $H p$. As the $H$ is found, fre- 
quency channel response. Then, the ML of the transmitted signal can be expressed as

$$
\bar{X}(n)=\arg \min _{X \in M}|Y(n)-H(n) X(n)|^{2}, 0 \leq n \leq 2 N-1
$$

The final estimation of the transmitted signal, $\mathrm{X}$, can be found by

$$
X(n)=\text { Quantization }\left(\frac{Y(n)}{H(n)}\right), 0 \leq n \leq 2 N-1
$$

Two factors that have a high impact on the performance of the EM-VLC algorithm are the number of iterations and the initialization step. Greater accuracy can be achieved with a greater number of iterations. However, as the number of iterations increases the complexity increases as well. Initialization can be performed either with pilot insertion or without pilots by using the statistical properties of the signal, such as in direct decision channel estimation. In this paper, we use LS estimator pilot insertion for initialization, using a grid-type pilot as in [8] for a more appropriate initial value for $\bar{X}_{0}$.

It is possible to obtain results that are more accurate with pilot insertion [38], and less time is required for convergence. On the other hand, a direct decision is undesirable in real-time estimation because the data detection of the current estimation relies on the previous OFDM symbol, then the new detected data is used for the estimation of the current channel [39]. Practically, the EM-VLC algorithm is a highly robust technique for channel estimation when the available data are incomplete. This is because it has high accuracy and less complexity compared to other channel estimation methods.

\section{Simulation results and discussion}

\subsection{Simulation setup}

The optical channel with IM/DD in the VLC was modelled as a baseband linear system [40] with the channel response that was defined above in Equation (2). In the simulation setup, linear scaling and DC bias were used so that the OFDM signal was within the dynamic range and non-negativity was constrained, respectively. This setup is called DCO-OFDM. The transmitted bits were randomly generated and M-ary quadrature amplitude modulation (M-QAM) symbols were formed by serial to parallel conversion. The QAM symbols were assigned to subcarriers. Thus, the light source was a modulated signal that could be transmitted over the OWC channel. At the receiver, the reverse process was executed after the DC bias as removed. The VLC channel is not subject to fast fading effects because it uses a very small wavelength compared to the detector area [41]. However, it is subject to slow fading, which is the result of shadowing from furnishings or other indoor objects. The effect of shadowing can be overcome by increasing the number of LEDs. The received optical signal power, distributed on the receiver plane was estimated based on the lineof-sight channel model and by ignoring the reflection off the walls. The simulation parameters are provided in Table 1.

Table 1: Simulation Parameters

\begin{tabular}{ll}
\hline Parameter & Specification \\
\hline System & DCO-OFDM-based VLC \\
IFFT size (N) & $16,32,64,128,512$ \\
Channel & OWC with WGN \\
Modulation schemes & 16,64 QAM \\
Pilot insertion type & Grid type \\
Pilot spacing & $4,6,8$ \\
Initial channel estimation & LS estimator \\
\hline
\end{tabular}

\subsection{Results and discussion}

In this section, a comprehensive analysis of the performance of the proposed EM-VLC algorithm for OFDM VLC channel estimation is presented. Figure 2 shows the BER produced by the proposed algorithm when using a FFT size of 64 and a pilot spacing of 8 . The proposed algorithm performs better than the other three channel estimation methods, LS, MMSE and Ir-MMSE. This is due to the fast convergence of the EM-VLC algorithm. A comparatively low BER is achieved by the EM-VLC algorithm at different SNR values. As explained in section 4 , and based on the analytical results, the EM-VLC algorithm has less complexity than the MMSE channel estimation method because the former can estimate unknown parameters (noise variance and channel covariance) and retrieve transmitted bits.

The MMSE estimator requires prior knowledge of the channel covariance and noise variance. Furthermore, the MMSE estimator has higher complexity than the LS estimator. However, the LS estimator is less accurate than the MMSE estimator. Thus, the higher the SNR, the better the performance of the MMSE estimator in comparison to the LS estimator.

On the other hand, the enhanced lr-MMSE estimator is a modified MMSE estimator and it has less complexity due to some simplifications, as explained in section 3.2.3. Thus this estimator is, in essence, a compromise between the MMSE and LS estimators in respect of performance and complexity. Therefore, the BER of the lr-MMSE estimator lies between that of the MMSE and LS estimators. The worst BER occurs when no channel estimation method is applied.

In this paper, the highest BER at all SNR values occurs when no channel estimation is used, while the lowest BER at all SNR values occurs when the EM-VLC algorithm is used. For example, at a SNR of $13 \mathrm{~dB}$, the proposed EM-VLC algorithm has a BER of 101. This value shows a gain of [1], [2]. [5] And [3] dB of BER over the other channel estimation methods, MMSE, 1r-MMSE and LS, respectively.

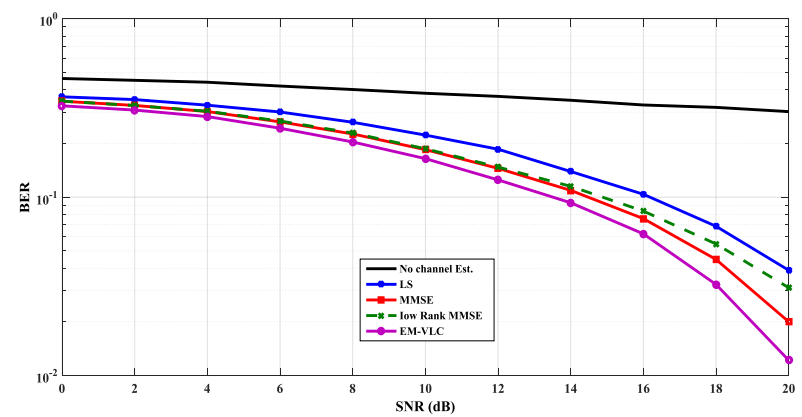

Fig. 2: BER Performance of EM-VLC Algorithm for 64 FFT and Pilot Spacing of [8].

Figures 3 and 4 provide the results of a comparison of the BER of the proposed EM-VLC algorithm with that of the LS, MMSE and lr-MMSE algorithms, in which six and four pilot insertions were used, respectively. The results show that increasing the pilot density (i.e., less pilot spacing) can improve estimation accuracy. However, this introduces a lower transmitted bit rate and results in poorer spectral efficiency. Moreover, the pilot arrangement or pilot pattern, which is a grid type in this paper, can also affect channel estimation. Hence, an optimum pilot arrangement is a trade-off between accuracy and the energy wasted [29].

It is clear from Fig. 3 and Fig. 4 that, in the proposed method, the BER is maintained at almost the same value when the pilot spacing is decreased. This is because pilot insertion is only used to find the initial value. Therefore, unnecessary pilot insertions can be avoided when using the EM-VLC algorithm, which improves the transmitted bit rate and results in higher spectral efficiency. The other estimators need a high number of pilots to track the channel changes for better channel estimation, which is especially the case for the MMSE estimator. In the proposed method, computational complexity is significantly reduced because complexity is extremely dependent on the number of subcarriers.

For instance, in Fig. 3, the EM-VLC algorithm produces a lower BER compared to the other three algorithms. For instance, it has a BER of 10-1 at a SNR of $12 \mathrm{~dB}$, whereas MMSE, lr-MMSE and 
LS need have a higher SNR of 14.5, 15 and $16.5 \mathrm{~dB}$, respectively, in order to produce a similar BER. In Fig. 4, the number of pilots is higher (i.e., there is less pilot spacing), so the BER is higher. For example, the EM-VLC algorithm achieves a BER of $10^{-1}$ at 12 $\mathrm{dB}$, whereas MMSE, lr-MMSE and LS need to have a higher SNR of $15,15.5$ and $17 \mathrm{~dB}$, respectively, in order to produce a similar BER. This is because the fewer the pilot insertions, the higher the bit rate that can be transmitted.

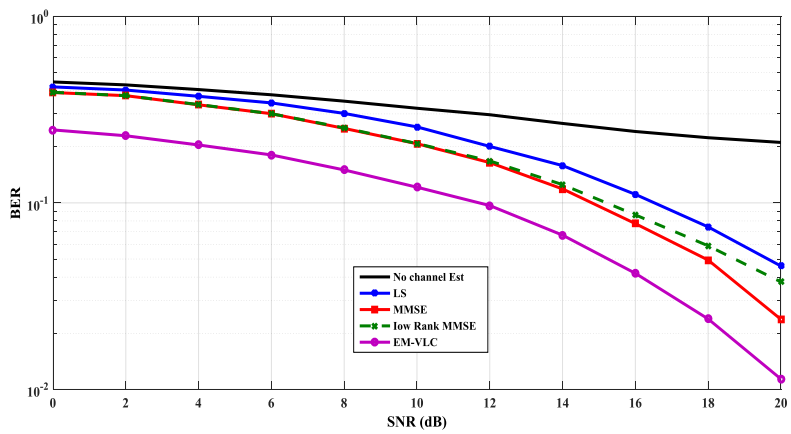

Fig. 3: Comparative BER Performance for 64 FFT and Pilot Spacing of [6].

To evaluate the effectiveness of the proposed EM-VLC algorithm for OFDM VLC systems, several simulations were carried out for different FFT sizes and modulation schemes. Figure 5 shows that the proposed EM-VLC algorithm produces a lower BER than the MMSE, lr-MMSE and LS estimators when the FFT size increases.

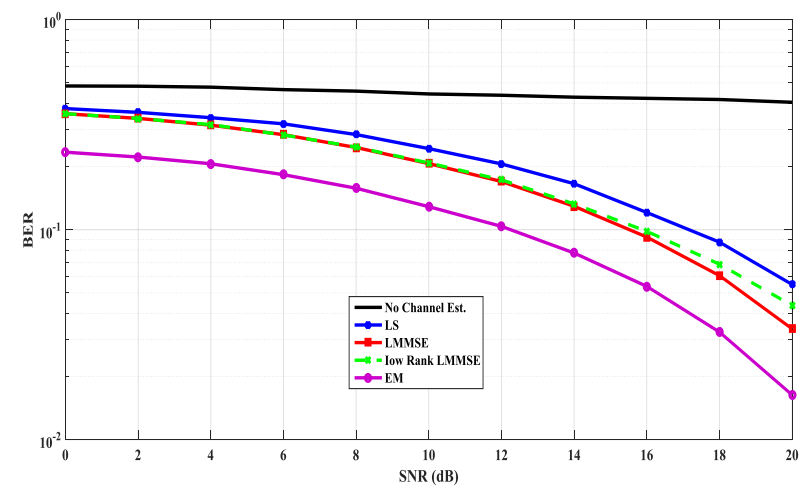

Fig. 4: Comparative BER Performance for 64 FFT and Pilot Spacing of [4].

In Figure 5, the EM-VLC algorithm has a BER of $10^{-1}$ at a SNR of $13 \mathrm{~dB}$ with a FFT size of 512 , which is the same as the BER and SNR it produced at the FFT size of 64 (see Fig. 2). The MMSE, lrMMSE and LS estimators at a BER of $10^{-1}$ achieve about a 0.9 , 1.7 and $0.2 \mathrm{~dB}$ reduction in SNR, respectively, with a FFT size of 512, compared with a FFT size of 64 in Fig. 2. In other words, the proposed EM-VLC algorithm can produce the best BER at all FFT sizes.

However, the other estimators (MMSE, lr-MMSE and LS) do perform better when the FFT size is larger (for example, 512 FFT compared to 64 FFT). This is because when the FFT size is increased, more samples are added for each signal. Consequently, the signal is smoother and more accurate.

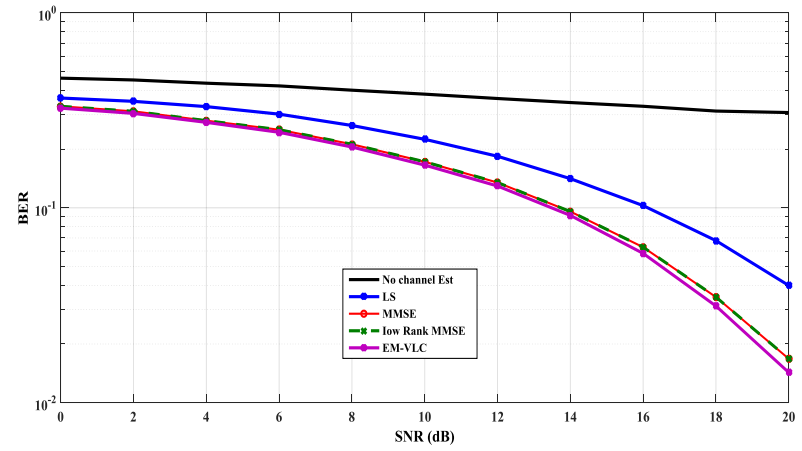

Fig. 5: Comparative BER Performance for 512 FFT and Pilot Spacing of [4].

Figure 6 shows that the proposed EM-VLC algorithm produces almost the same BER at FFT sizes ranging from 16 to 256. This indicates that the proposed method has another significant advantage, i.e.,the accuracy of the EM-VLC estimator is independent of the increase in the number of samples for each signal. In addition, the EM-VLC algorithm maintains the lowest BER compared to all the other estimators (MMSE, lr-MMSE and LS) at different FFT sizes because it does not rely on a large number of pilots because it only uses pilots at initialization.

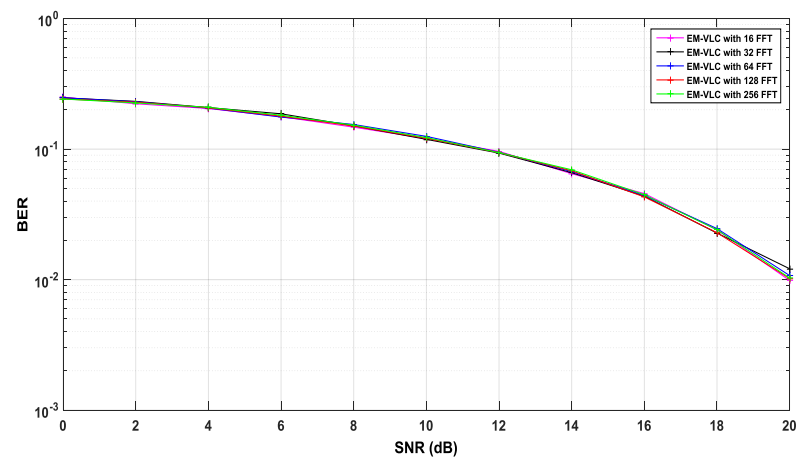

Fig. 6: BER of EM-VLC Algorithm with 64 QAM at Different FFT.

Figure 7 depicts the BER produced by the compared estimators at different QAM. Obviously, the BER decreases gradually as the SNR increases for all types of QAM. As can be seen from Fig. 7, the proposed EM-VLC algorithm outperforms all of the other channel estimation methods for all types of QAM. This stems from the EM-VLC algorithm's ability to estimate unknown parameters in a very low number of iterations before convergence.

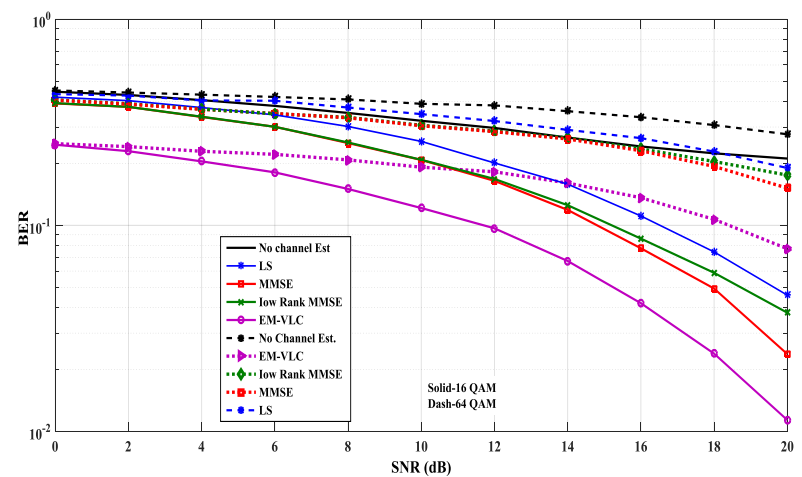

Fig. 7: Comparative BER Performance with 16 QAM and 64 QAM.

It is also clear from Fig. 7 that the BER increases when the QAM order increases for each channel estimation algorithm. For instance, with 16-QAM the performance of the MMSE estimator is better than that of the LS estimator; the MMSE estimator can achieve the same BER of $10^{-1}$ but at a lower SNR of $3 \mathrm{~dB}$. In other words, the higher the SNR, the better the performance of the MMSE estimator compared to the LS estimator. Moreover, the LS estimator is susceptible to channel noise and variance in the esti- 
mation error. Indeed, the performance of the LS estimator is highly dependent on the noise level. On the other hand, the MMSE estimator is more powerful in terms of its ability to eliminate noise and because it relies on channel frequency correlation it obtains a better estimation. However, it does have higher complexity than the LS estimator. As mentioned above, the lr-MMSE estimator developed in this paper is a compromise between the MMSE and LS estimators in terms of performance and complexity. Hence it produces a better BER than the MMSE, except at higher SNR, and better BER than the LS estimator. In light of the above, the performance of the proposed EM-VLC algorithm is superior to that of all the other methods compared in this paper.

\section{Conclusion}

The receiver of an OFDM-based VLC system needs to be equipped with high-precision channel estimation to improve data detection performance. Therefore, in this paper, a new EM-VLC channel estimation algorithm for OFDM-based VLC systems was proposed and evaluated. In addition, a low-rank minimum mean square error channel estimation algorithm (lr-MMSE) was developed and assessed. The proposed EM-VLC algorithm is an iterative method for computing the ML channel estimation and finding unknown parameters. It was evaluated and compared with three different channel estimation methods, MMSE, lr-MMSE and LS, as well as with no channel estimation. Extensive simulations were conducted to assess the robustness of the proposed algorithm.

The results demonstrated that the proposed EM-VLC algorithm produced a much lower BER and had less complexity than the MMSE, Ir-MMSE and LS channel estimation methods. This is because EM-VLC is not dependent on pilot insertion, except at initialization, and yet this independence does not affect its ability to find unknown parameters. While the LS estimator is simple it produces a higher BER than MMSE. On the other hand, although the MMSE estimator produces a lower BER than LS, it has higher complexity. The lr-MMSE estimator was developed in this study as a compromise between the MMSE and LS estimators in order to achieve a better balance between performance and complexity. Thus, in practice, the proposed EM-VLC algorithm provides a great trade-off between accuracy and complexity. In addition, it is more suitable for real-time estimation. In future work, the EMVLC algorithm could be extended to make it suitable for a more sophisticated OFDM-based VLC system, such as multiple-input multiple-output (MIMO).

\section{References}

[1] Singh, S., \& Bharti, R. (2014).163m/10Gbps 4QAM-OFDM visible light communication. IJETR, 2, 225-228.

[2] Burchardt, H., Serafimovski, N., Tsonev, D., Videv, S., \& Haas, H. (2014). VLC: Beyond point-to-point communication. Communications Magazine, IEEE, 52(7), 98-105. https://doi.org/10.1109/MCOM.2014.6852089.

[3] Li, Y., Tsonev, D., \& Haas, H. Non-DC-biased OFDM with optical spatial modulation. In Personal Indoor and Mobile Radio Communications (PIMRC), 2013 IEEE 24th International Symposium on, 2013 (pp. 486-490): IEEE.

[4] Xia, C., Hainfeng, W., \& Honglin, H. A Low Complexity PilotAided Channel Estimation Resisting Residual Timing Errors for OFDM Systems. In Personal, Indoor and Mobile Radio Communications, 2007. PIMRC 2007. IEEE 18th International Symposium on, $\quad 3-7 \quad$ Sept. $2007 \quad 2007 \quad$ (pp. $1-5$ ) https://doi.org/10.1109/PIMRC.2007.4394644

[5] Guvenc, I., Gezici, S., Sahinoglu, Z., \& Kozat, U. C. (2011). Reliable communications for short-range wireless systems: Cambridge University Press. https://doi.org/10.1017/CBO9780511974366.

[6] Yang, X., Min, Z., Xiongyan, T., Jian, W., \& Dahai, H. A postprocessing channel estimation method for DCO-OFDM visible light communication. In Communication Systems, Networks \& Digital Signal Processing (CSNDSP), 2012 8th International Symposium on, 2012 (pp. 1-4): IEEE.

[7] Zhang, T., Guo, S., Chen, H., Zhong, F., \& Ma, C. (2014). Enhancing the bit error rate of indoor visible light communication systems using adaptive channel estimation algorithm. IET Communications, 9(4), 501-507. https://doi.org/10.1049/iet-com.2014.0649.

[8] Lin, W.-F., Chow, C.-W., \& Yeh, C.-H. (2015). Using specific and adaptive arrangement of grid-type pilot in channel estimation for white-lightLED-based OFDM visible light communication system.
Optics
Communications,
338
$7-10$ https://doi.org/10.1016/j.optcom.2014.09.080.

[9] Chen, X., \& Jiang, M. (2017). Adaptive Statistical Bayesian MMSE Channel Estimation for Visible Light Communication. IEEE Transactions on Signal Processing, 65(5), 1287-1299. https://doi.org/10.1109/TSP.2016.2630036.

[10] Wang, J.-b., Jiao, Y., Dang, X.-y., Chen, M., Xie, X.-X., \& Cao, L.-1. (2011). Training sequence based channel estimation for indoor visible light communication system. Optoelectronics Letters, 7, 213 216. https://doi.org/10.1007/s11801-011-0143-7.

[11] Wang, J.-B., Jiao, Y., Song, X., \& Chen, M. (2011). Optimal training sequences for indoor wireless optical communications. Journal of Optics, 14(1), 015401. https://doi.org/10.1088/20408978/14/1/015401

[12] Wang, J.-B., Jiao, Y., Song, X., Zhao, X., Gu, M., Chen, M., et al (2011). Complementary sequences-based channel estimation for diffuse wireless optical communications. Optical Engineering, 50(7), 075003-075003-075006.

[13] Pham, Q. N., Rachim, V. P., an, J., \& Chung, W.-Y. (2017). Ambient Light Rejection Using a Novel Average Voltage Tracking in Visible Light Communication System. Applied Sciences, 7(7), 670. https://doi.org/10.3390/app7070670.

[14] Wang, J.-B., Xie, X.-X., Jiao, Y., \& Chen, M. (2012). Training sequence based frequency-domain channel estimation for indoor diffuse wireless optical communications. EURASIP Journal on Wireless Communications and Networking, 2012(1), 1-10. https://doi.org/10.1186/1687-1499-2012-326.

[15] Zhuang, X., \& Vook, F. W. Iterative channel estimation and decoding for a turbo-coded OFDM system via the EM algorithm. In Acoustics, Speech, and Signal Processing (ICASSP), 2002 IEEE International Conference on, 2002 (Vol. 3, pp. III-2337-III-2340): IEEE

[16] Krishna, E. H., Sivani, K., \& Reddy, K. A. OFDM channel estimation and equalization using multi scale independent component analysis. In Signal Processing, Informatics, Communication and Energy Systems (SPICES), 2015 IEEE International Conference on, 2015 (pp. 1-5): IEEE

[17] Popoola, W. O., Ghassemlooy, Z., \& Stewart, B. G. (2014). Pilotassisted PAPR reduction technique for optical OFDM communication systems. Journal of Lightwave Technology, 32(7), 1374-1382. https://doi.org/10.1109/JLT.2014.2304493.

[18] Popoola, W. O., Ogunkoya, F. B., \& Sinanović, S. Mitigating nonlinearities under average power constraint in visible light communication. In Wireless Communications and Mobile Computing Conference (IWCMC), 2014 International, 2014 (pp. 815-820): IEEE.

[19] Hussein, Y. S., Alias, M. Y., \& Abdulkafi, A. A. On performance analysis of LS and MMSE for channel estimation in VLC systems. In 2016 IEEE 12th International Colloquium on Signal Processing \& Its Applications (CSPA), 2016 (pp. 204-209): IEEE.

[20] Dissanayake, S. D., \& Armstrong, J. (2013). Comparison of acoofdm, dco-ofdm and ado-ofdm in im/dd systems. Journal of Light$\begin{array}{lll}\text { wave } & \text { Technology, } & \text { 31(7), }\end{array}$ https://doi.org/10.1109/JLT.2013.2241731.

[21] Ma, X., Kobayashi, H., \& Schwartz, S. C. (2004). EM-based channel estimation algorithms for OFDM. EURASIP Journal on Applied Signal Processing, 2004, 1460-1477.

[22] Karkhanechi, H. M., \& Levy, B. C. EM-based channel estimation for space-time block coded MIMO OFDM systems. In Signal Processing Systems Design and Implementation, 2006. SIPS'06. IEEE Workshop on, 2006 (pp. 177-181): IEEE

[23] Kabaoğlu, N. (2013). EM Based Data Detection Algorithm for Downlink of a Single Hop Relaying Network. Wireless Personal Communications, 1-14. https://doi.org/10.1007/s11277-012-0562-1.

[24] Al-Naffouri, T. Y. (2007). An EM-based forward-backward Kalman filter for the estimation of time-variant channels in OFDM. IEEE Transactions on Signal Processing, 55(7), 3924-3930. https://doi.org/10.1109/TSP.2007.894271

[25] Vizziello, A., Savazzi, P., \& Favalli, L. Mitigation of intercarrier interference in OFDM systems over slow fading channels based on the EM algorithm. In MELECON 2010-2010 15th IEEE Mediterranean Electrotechnical Conference, 2010 (pp. 1320-1325): IEEE.

[26] Armstrong, J., \& Schmidt, B. J. (2008). Comparison of asymmetrically clipped optical OFDM and DC-biased optical OFDM in AWGN. Communications Letters, IEEE, 12(5), 343-345. https://doi.org/10.1109/LCOMM.2008.080193. 
[27] Carruthers, J. B., \& Kahn, J. M. Modeling of nondirected wireless infrared channels. In Communications, 1996. ICC'96, Conference Record, Converging Technologies for Tomorrow's Applications. 1996 IEEE International Conference on, 1996 (Vol. 2, pp. $1227-$ 1231): IEEE

[28] Cho, Y. S., Kim, J., Yang, W. Y., \& Kang, C. G. (2010). MIMOOFDM wireless communications with MATLAB: John Wiley \& Sons. https://doi.org/10.1002/9780470825631.

[29] Ozdemir, M. K., \& Arslan, H. (2007). Channel estimation for wireless OFDM systems. IEEE Communications Surveys \& Tutorials, 9(2), 18-48. https://doi.org/10.1109/COMST.2007.382406.

[30] Bandara, K., Niroopan, P., \& Chung, Y.-H. PAPR reduced OFDM visible light communication using exponential nonlinear companding. In Microwaves, Communications, Antennas and Electronics Systems (COMCAS), 2013 IEEE International Conference on, 2013 (pp. 1-5): IEEE.

[31] Arslan, H., \& Bottomley, G. E. (2001). Channel estimation in narrowband wireless communication systems. Wireless Communications and Mobile Computing, 1(2), 201-219. https://doi.org/10.1002/wcm.14.

[32] Won Gi, J., Kyung Hyun, P., \& Yong Soo, C. An efficient channel estimation technique for OFDM systems with transmitter diversity. In Personal, Indoor and Mobile Radio Communications, 2000. PIMRC 2000. The 11th IEEE International Symposium on, 2000 2000 (Vol. 2, pp. 1246-1250 vol.1242). https://doi.org/10.1109/PIMRC.2000.881618.

[33] Wang, S., \& Abdi, A. (2005). Aperiodic complementary sets of sequences-based MIMO frequency selective channel estimation. IEEE Communications Letters, 9(10), 891-893. https://doi.org/10.1109/LCOMM.2005.10019.

[34] Shen, Y., \& Martinez, E. (2006). Channel estimation in OFDM systems. Application note, Freescale semiconductor.

[35] Carpentier, A., \& Kim, A. K. (2015). An iterative hard thresholding estimator for low rank matrix recovery with explicit limiting distribution. arXiv preprint arXiv:1502.04654.

[36] Ma, X., Kobayashi, H., \& Schwartz, S. C. An EM-based estimation of OFDM signals. In Wireless Communications and Networking Conference, 2002. WCNC2002. 2002 IEEE, 2002 (Vol. 1, pp. 228 232): IEEE.

[37] Moon, T. K. (1996). The expectation-maximization algorithm. Signal processing magazine, IEEE, 13(6), 47-60. https://doi.org/10.1109/79.543975.

[38] Xie, Y., \& Georghiades, C. N. (2003). Two EM-type channel estimation algorithms for OFDM with transmitter diversity. Communications, IEEE Transactions on, 51(1), 106-115, https://doi.org/10.1109/TCOMM.2002.807617.

[39] Bossert, M., Donder, A., \& Trushkin, A. (1995). Channel estimation and equalization in orthogonal frequency division multiplexing systems. ITG FACHBERICHT, 485-485.

[40] González, O., Guerra, M., \& Martín, R. (2014). Multi-User Visible Light Communications. Advances in Optical Communications, 3663.

[41] Wang, C.-X., Haider, F., GAO, X., You, X.-H., Yang, Y., Yuan, D., et al. (2014). Cellular architecture and key technologies for 5G wireless communication networks. IEEE Communications magazine, 52(2), 122-130. https://doi.org/10.1109/MCOM.2014.6736752. 